\title{
Cataclysmic Variables - X-rays and Optical Activity in V1223 Sgr and V709 Cas
}

\author{
R. Gális, L. Hric, E. Kundra, F. Münz
}

\begin{abstract}
Intermediate polars are a major fraction of all cataclysmic variables detected by INTEGRAL in hard X-ray. These objects have recently been proposed to be the dominant X-ray source population detected near the Galactic centre, and they also contribute significantly to X-ray diffuse Galactic ridge emission. Nevertheless, only $25 \%$ of all known intermediate polars have been detected in hard X-ray. This fact can be related to the activity state of these close interacting binaries. A multi-frequency (from optical to X-ray) investigation of intermediate polars is essential for understanding the physical mechanisms responsible for the observed activity of these objects.
\end{abstract}

Keywords: Cataclysmic variables, optical and X-ray variability, mass transfer and accretion.

\section{Introduction}

Cataclysmic variables (CVs) manifest strong activity in a whole spectrum from radio up to $\gamma$-rays. CVs are close binary systems consisting of a hot white dwarf (WD) and a red main-sequence star of spectral type $\mathrm{M}$ or $\mathrm{K}$, which fills the volume of its inner Roche lobe and transfers matter to the vicinity of the WD [1]. The mass transfer between components causes the observed activity of the $\mathrm{CVs}$, which varies from relatively small light variations (flickering) to enormous photometric changes (outbursts of novae) on time scales that cover a wide range from very fast variability of fractions of seconds (flickering) to longterm variations of several years or decades (activity cycles).

According to strength of the WD magnetic field, the transferred matter creates an accretion disk (classical CVs) or follows magnetic lines and falls to the surface of the WD (magnetic CVs). Magnetic CVs are a small sub-set of all catalogued CV systems, and fall into two categories: polars and intermediate polars (IPs). In IPs, the WD magnetic field $\left(10^{6}-10^{7} \mathrm{G}\right)$ is not strong enough to disrupt the disc entirely (as in the case of polars) and it simply truncates the inner part of the disc [2]. An accretion flow is channelled down towards the magnetic poles and onto the WD surface.

Increased interest in CVs was aroused after the discovery of the very hard X-ray emission (up to $80 \mathrm{keV}$ ) of many IPs. When the transferred material impacts the WD atmosphere, a strong shock will form above its surface [3]. The temperature in the post-shock region (PSR) can be very high and the plasma is cooled mainly via optically thin bremsstrahlung radiation in the hard X-ray band [4].
As we showed in our previous analysis [5], the broadband spectra $(3-100 \mathrm{keV})$ of the studied IPs can be well fitted by a thermal bremsstrahlung model with PSR temperature $k T \approx(20-25) \mathrm{keV}$. Reflection of the bremsstrahlung photons at an optically thick cold medium can also contribute to the hard X-ray spectrum [6]. In the case of V2400 Oph, a significant emission excess detected at $\approx 26 \mathrm{keV}$ can be caused by the reflection of $\mathrm{X}$-rays from the surface of the WD [5].

In total, $32 \mathrm{CVs}$ (and 2 symbiotic systems) were detected in the data of INTEGRAL $[7-9,5]$. This is more than was expected, and it represents $\approx 5 \%$ of all detections of this space observatory. The sample of IPs detected in the (20-40) keV energy band has 23 members, representing more than $70 \%$ of all $\mathrm{CVs}$ detected by INTEGRAL [5]. IPs are the most luminous and the hardest $\mathrm{X}$-ray sources among accreting WDs. In hard X-rays, these objects seem to be more luminous (up to a factor of 10) than polars [10]. In strongly magnetized $\left(B \geq 10^{7} \mathrm{G}\right)$ polar systems, cyclotron radiation is an important cooling mechanism, which suppresses high temperature bremsstrahlung emission, whilst it should be negligible for IPs. This could explain why most of the CVs observed in the hard X-ray band are IPs.

Short-term soft X-ray modulations have been observed in the orbital period, in the spin period of the WD, or a beat between the two. No significant modulation has been found so far in the $20-30 \mathrm{keV}$ light curves [11]. Most CVs seem to have persistent soft gamma ray fluxes. Nevertheless, the sample detected by INTEGRAL represents only $25 \%$ of all known IPs [5]. Some IPs are not detectable even if we have significant exposure time (more than 4Msec) for these sources. This fact can be related to the activ- 

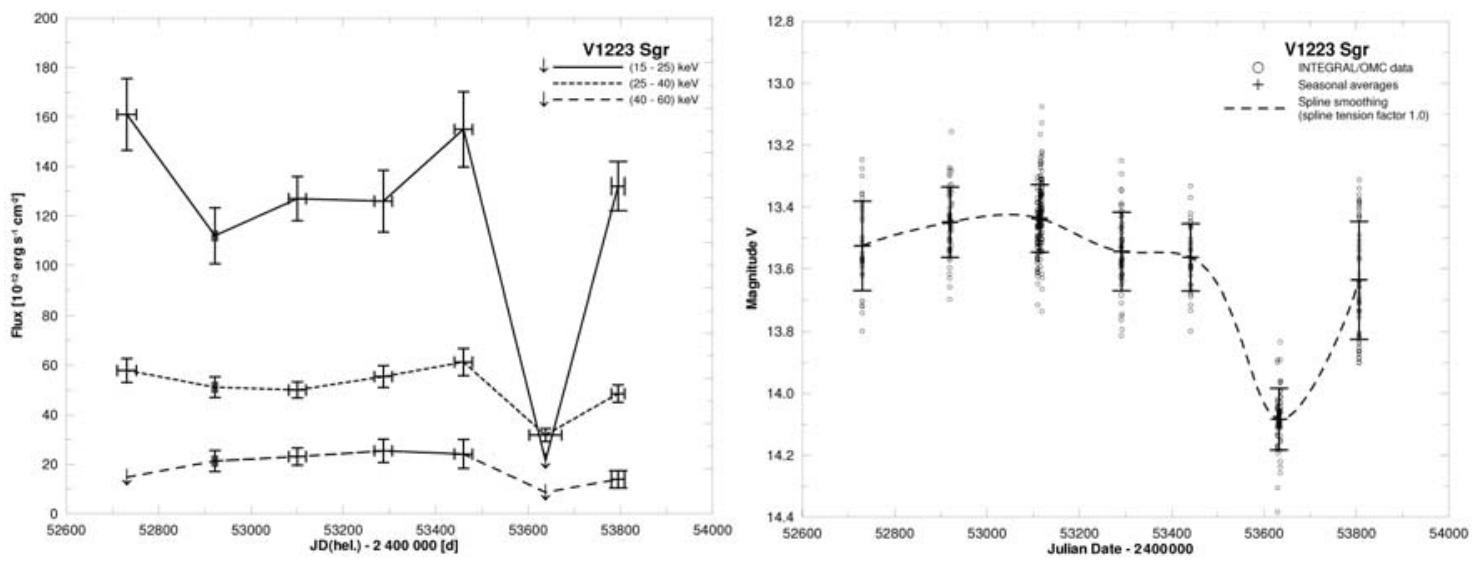

Fig. 1: Left panel: INTEGRAL/IBIS flux curves of V1223 Sgr in the corresponding energy bands. The arrows represent $3 \sigma$ upper limits. Right panel: INTEGRAL/OMC light curve of V1223 Sgr in the optical spectral band. The crosses represent average magnitude values in the corresponding seasons

ity state of these interacting binaries. To understand this relation, it is necessary to study the correlation between the activity state and the X-ray emission of IPs.

\section{Observations, analysis and results}

We used all publicly available observational data from INTEGRAL/JEM-X and INTEGRAL/IBIS to study possible variability of the selected IPs in the hard X-ray and soft $\gamma$-ray spectral bands. In addition, observations from INTEGRAL/OMC data were used to look for long-term variability of these objects in optical band. The observational data used in our analysis was processed by INTEGRAL's Offline Standard Analysis Package OSA7. The libraries grid.py and ms_suite.py developed by FM were also used for the data processing and preparation of the final mosaics.

\subsection{Intermediate polar V1223 Sgr}

IP V1223 Sgr is a bright X-ray source (4U 1849-31) with possible X-ray flare activity. A short-term burst has also been detected from this system in the optical [12]. These outbursts are probably a result of disk instabilities or an increase in mass transfer, but there is no correlation between optical and X-ray burst activity. Moreover, episodes of deep low state (decrease by several magnitudes) of V1223 Sgr in the optical band have also been detected [13].

The observational material for IP V1223 Sgr consisted of 132 (INTEGRAL/JEM-X) and 1375 (INTEGRAL/IBIS) individual pointings obtained in the course of almost three years (MJD 52710.3853809.25). The overall INTEGRAL/IBIS mosaics (total exposure time $1405.5 \mathrm{ksec}$ ) showed that V 1223 Sgr is detectable up to $80 \mathrm{keV}$ with $\approx 5 \sigma$ significance. The broad-band (3-100) keV spectrum was very well fitted by a bremsstrahlung model with temperature $k T=23.7_{-1.3}^{+1.4} \mathrm{keV}[5]$.

During the monitored period, the mean fluxes of this object were $(103.0 \pm 4.0) \times 10^{-12} \mathrm{erg} \mathrm{cm}^{-2} \mathrm{~s}^{-1}$, $(46.4 \pm 1.4) \times 10^{-12} \mathrm{erg} \mathrm{cm}^{-2} \mathrm{~s}^{-1}$, $(15.1 \pm 1.5) \times 10^{-12} \mathrm{erg} \mathrm{cm}^{-2} \mathrm{~s}^{-1}$ and $(12.3 \pm 2.0) \times 10^{-12} \mathrm{erg} \mathrm{cm}^{-2} \mathrm{~s}^{-1}$ in $(15-25) \mathrm{keV}$, (25-40) keV, (40-60) keV and (60-80) keV band, respectively.

The inspection of the data showed that the observations were obtained in the course of 7 separate seasons. As the next step we split the data according these seasons to investigate long-term X-ray $/ \gamma$-ray variability. The flux curves are displayed in Figure 1 (left panel). It is clear that during the monitored period the fluxes of V1223 Sgr were long-term variable (especially in the softer bands), with a significant drop around MJD $\approx 53650$.

The optical light curve of V1223Sgr (INTE$G R A L / \mathrm{OMC}$ ) is shown in Figure 1 (right panel). We can see that optical brightness of this source was long-term variable, too. Moreover, these light variations are strongly correlated with the changes in the (15-25) keV, (25-40) keV and (40-60) keV spectral bands with correlation coefficients $0.81,0.82$ and 0.89 , respectively.

Our detailed period analysis of INTEGRAL/ OMC data did not yield any significant period, only partial detection of the orbital period $P_{\text {orb }}=$ $3.37 \mathrm{hrs}$ [14]. This was probably caused by complex intrinsic variability (flickering) of V1223 Sgr, possible period variations or a the drastic change in the brightness of this object during monitored period. However, a particular analysis is not possible due to inappropriate time distribution of the data. 

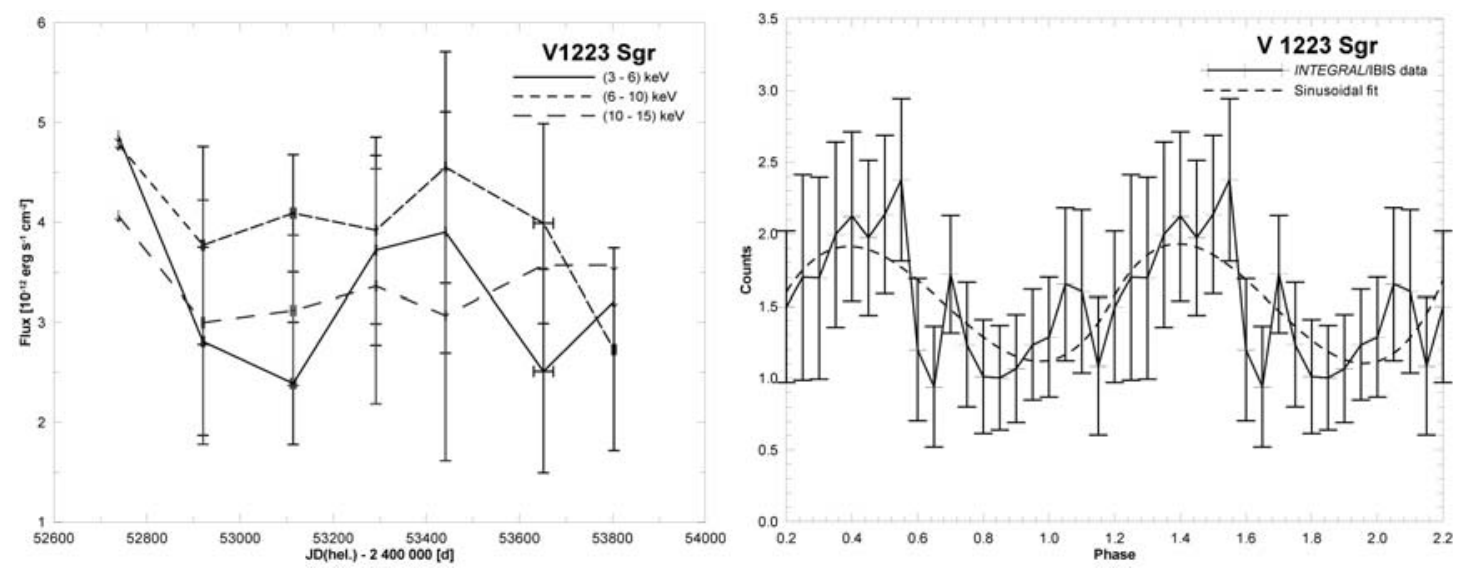

Fig. 2: Left panel: INTEGRAL/JEM-X flux curves of V $1223 \mathrm{Sgr}$ in the corresponding energy bands. The arrows represent $3 \sigma$ upper limits. Right panel: INTEGRAL/IBIS phase diagram of V1223 Sgr in (15-25) keV band folded with orbital period $(3.37 \mathrm{hrs})$

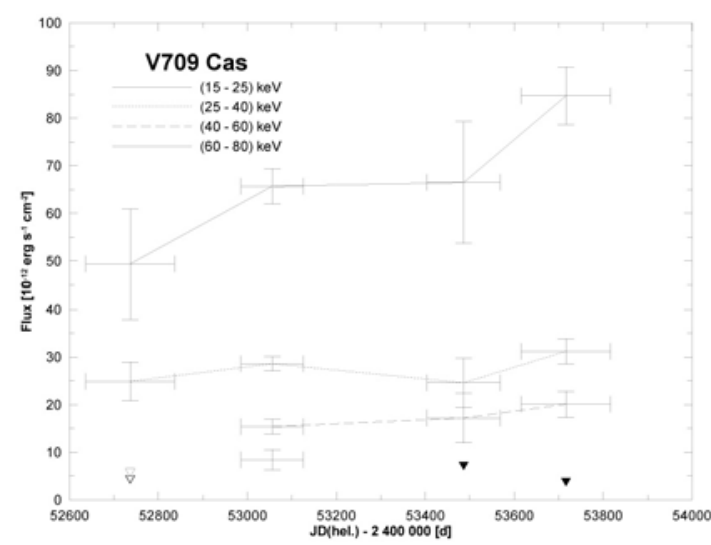

Fig. 3: INTEGRAL/IBIS light curves of V709 Cas in the corresponding energy bands. The arrows represent $3 \sigma$ upper limits

We also prepared overall mosaics using all available data from INTEGRAL/JEM- X. The total exposure time was $80.6 \mathrm{ksec}$. The medial fluxes of V1223 Sgr during the monitored period were $(2.8 \pm 0.4) \times 10^{-12} \mathrm{erg} \mathrm{cm}^{-2} \mathrm{~s}^{-1},(3.7 \pm 0.3) \times$ $10^{-12} \mathrm{erg} \mathrm{cm}^{-2} \mathrm{~s}^{-1},(2.6 \pm 0.4) \times 10^{-12} \mathrm{erg} \mathrm{cm}^{-2} \mathrm{~s}^{-1}$ and $(4.9 \pm 1.1) \times 10^{-12} \mathrm{erg} \mathrm{cm}^{-2} \mathrm{~s}^{-1}$ in $(3-6) \mathrm{keV}$, (6-10) $\mathrm{keV},(10-15) \mathrm{keV}$ and $(15-25) \mathrm{keV}$ band, respectively. We also spit the data to 7 seasons to investigate the long-term X-ray variability of V1223 Sgr. The flux curves are displayed in Figure 2 (left panel). As we can see the corresponding errors are too large for subsequent analysis and therefore we can conclude that the INTEGRAL/JEM-X fluxes of V1223 Sgr were persistent within their errors in the monitored period.

Typically, soft X-ray modulations were observed in the orbital period, in spin period of WD, or a beat between the two in IPs. However, the IPs are close binary systems with orbital periods in the order of hours and these objects, are not detectable on these time scales by INTEGRAL/IBIS. As the next step, we attempted to investigate the possible short-term variability of V1223 Sgr in INTEGRAL/IBIS data. We prepared a unique method of folding the particular phase interval on the basis of proper time intervals from the individual science windows. Our method applied Good Time Intervals (GTIs) according to the (orbital or other) phase bin and created phase resolved mosaics (assuming sufficient exposure) of a periodic source. A phase diagram of the fluxes V1223 Sgr in the (15-25) keV band folded with the orbital period and constructed using the data from time interval MJD (52917.17-52926.84) is shown in Figure 2 (right panel).

\subsection{Intermediate polar V709 Cas}

This X-ray source was recognized as an IP after it had been detected in the ROSAT All Sky Survey as RX J0028.8+5917 and was identified with a 14th magnitude blue star, V709 Cas. The broadband spectrum $(3-100) \mathrm{keV}$ of this object was fitted by the bremsstrahlung model with temperature $k T=29.6 \pm 2.5 \mathrm{keV}$, covering factor $C_{\mathrm{F}}=0.31 \pm 0.04$, column density $n_{\mathrm{H}}=(58 \pm 15) \times 10^{22} \mathrm{~cm}^{-2}$ and iron line energy $6.5 \pm 0.2 \mathrm{keV}$ [15]. The analysis showed that accounting for Compton scattering does not significantly change the obtained mass of the WD $\left(M_{\mathrm{WD}} \approx 0.9 M_{\odot}\right)$ in the case of V709 Cas [15]. The broad-band (3-100) keV spectrum from INTEGRAL was well fitted by a thermal bremsstrahlung model with post-shock temperature $k T=24.4_{-1.4}^{+1.5} \mathrm{keV}$ [5].

Our analysis of all available observational data for V709 Cas showed that this source is detectable up to $100 \mathrm{keV}$. The hard X-ray/soft $\gamma$-ray fluxes are not persistent, and the flux curves indicate that the brightness of this IP increased by a factor of $\approx 2$ from MJD 52700 to MJD 53700 in $(15-25) \mathrm{keV}$ energy band (Figure 3). 


\section{Conclusions}

We analysed all available observational data from $I N$ TEGRAL for IP V1223 Sgr and V709 Cas. Our analysis of the data from INTEGRAL/IBIS showed that the fluxes of these objects are long-term variable, mainly in the (15-25) keV and (25-40) keV bands. Moreover this hard X-ray/soft $\gamma$-ray variability is correlated with the changes in the optical spectral band in the case of V $1223 \mathrm{Sgr}$. Our analysis revealed a deep flux drop around MJD $\approx 53650$ observed in both the X-ray band and the optical band for this intermediate polar.

A significant part of the optical emission from IPs is produced by a hot spot, where the matter from a donor star interacts with the outer rim of the accretion disk. X-ray emission is produced by the interaction of the accreting matter with the WD surface. The emission in both the optical and the X-ray bands is therefore related to the mass transfer, and the observed variations are therefore probably caused by changes in the mass accretion rate.

We are preparing a photometric campaign to obtain long-term homogeneous observations (to cover whole activity cycles) as well as sets of observations with high time resolution (to cover the orbital cycles in detail) of selected CVs, mainly as a follow up to the INTEGRAL observations. Simultaneous analysis of multi-frequency observation (from optical to X-ray) enables a complex study of the physical mechanism related to the mass transfer in these interacting binaries.

\section{Acknowledgement}

The International Gamma-Ray Astrophysics Observatory (INTEGRAL) is a European Space Agency mission with instruments and a science data centre funded by the ESA member states (especially by the PI countries: Denmark, France, Germany, Italy, Spain, Switzerland), the Czech Republic and Poland, and with the participation of Russia and the USA. This study was supported by a project of the Slovak Academy of Sciences, VEGA Grant No. 2/0078/10.

\section{References}

[1] Warner, B.: Cataclysmic variable stars. Cambridge : Cambridge University Press, 1995.

[2] Patterson, J.: Publ. Astron. Soc. Pacific, 1994, 106, 209.

[3] Aizu, K.: Prog. Theor. Phys. 1973, 49, 1184.
[4] King, A. R., Lasota, J. P.: Mon. Not. R. Astron. Soc. 1979, 188, 653.

[5] Gális, R., Eckert, D., Paltani, S., Münz, F., Kocka, M.: Baltic Astronomy, 2009, 18, 321.

[6] van Teeseling, A., Kaastra, J. S., Heise, J.: Astron. Astrophys. 1996, 312, 186.

[7] Bird, A. J., Malizia, A., Bazzano, A., Barlow, E. J., et al.: Astrophys. J. Suppl. Ser. 2007, 170, 175 .

[8] Kniazev, A., Revnivtsev, M., Sazonov, S., Burenin, R., Tekola, A.: The Astronomer's Telegram, 2008, 1488.

[9] Masetti, N., Parisi, P., Palazzi, E., Landi, R., et al.: The Astronomer's Telegram, 2008, 1620.

[10] Chanmugam, G., Ray, A., Singh, K. P.: Astrophys. J. 1991, 375, 600.

[11] Barlow, E. J., Knigge, C., Bird, A. J., Dean, A., et al.: Mon. Not. R. Astron. Soc. 2006, 372, 224 .

[12] van Amerongen, S., van Paradijs, J.: Astron. Astrophys. 1989, 219, 195.

[13] Garnavich, P., Szkody, P.: Publ. Astron. Soc. Pacific, 1988, 100, 1522.

[14] Jablonski, F., Steiner, J. E.: Astrophys. J. 1987, 323, 672.

[15] Suleimanov, V., Poutanen, J., Falanga, M., Werner, K.: Astron. Astrophys. 2008, 491, 525.

R. Gális

Department of Theoretical Physics and Astrophysics Institute of Physics

Faculty of Sciences

P. J. Šafárik University

Park Angelinum 9, 04001 Košice, Slovakia

L. Hric

E. Kundra

Astronomical Institute

of the Slovak Academy of Sciences

05960 Tatranská Lomnica, Slovakia

F. Münz

Faculty of Science

Masaryk University

Kotlářská 2, 61137 Brno, Czech Republic 